\title{
Finite Element Analysis of Gradient Coil Deformation and Vibration in NMR Microscopy
}

\author{
W. C. Chu, C. B. Ahn, Member, IEEE, B. Bavarian, Member, IEEE, Sherif A. Mourad, and Russell E. Jacobs
}

\begin{abstract}
Resolution degradation due to gradient coil deformation and vibration in NMR microscopy is investigated using finite element analysis. From the analysis, deformations due to Lorentz's force can be as large as 1-10 $\mu \mathrm{m}$ depending upon the gradient strength and coil frame material. Thus, these deformations can be one of the major resolution limiting factors in NMR microscopy. Coil vibration, which depend on the input current waveform, and resolution degradation due to time-variant deformation and time-invariant deformation are investigated by numerical simulations.
\end{abstract}

\section{INTRODUCTION}

N NMR microscopy, high resolution images are obtained through the use of a high main magnetic field and high gradient fields [1], [2]. Three-dimensional gradient fields are usually generated using different shapes of gradient coils. For example, the $z$-directional gradient coil is made using a Maxwell pair of two opposite circular current loops. For the $x$ and $y$ gradient coils, four saddle-shaped coils are commonly used. When these coils are placed inside a strong magnetic field, large forces are induced according to the Lorentz equation, $F=I l \times B$ where $I$ is the current running in the coil, $l$ is the coil length in vector quantity, and $B$ is the main magnetic field. It is easily seen that the induced forces for the $z$ coil have only axial components pointing toward and away from the coil. For the $x-y$ Golay coils, the net forces on each of the middle four arcs surrounding the imagining volume have the same direction, $(+x$ or $-x$ direction for the $x$ gradient coil), while net forces on the outermost four arcs have the opposite direction, thus in an exaggerate fashion, they make the coil frame bend like an arch.

The induced forces generate stresses on the coil frame, which in turn cause deformations in the frame body. The magnitude of the deformations depend on the magnitude of the induced forces acting on the frame, as well as the material properties of the coil frames. In this paper, the coil frame deformations are calculated using the finite element method (FEM) [3], [4]. Based on this calculation, the effects of the

Manuscript received September 22, 1991; revised January 14, 1992. This work was supported in part by the NIH under Grant HD 25390. R. E. Jacobs is an Established Investigator of the American Heart Association.

W. C. Chu and B. Bavarian are with the Department of Electrical and Computer Engineering, University of California, Irvine, CA 92717.

C. B. Ahn is with the Department of Radiological Sciences, University of California, Irvine, CA 92717.

S. A. Mourad is with the Department of Civil Engineering, University of California, Irvine, CA 92717.

R. E. Jacobs is with the Department of Physiology and Biophysics, University of California, Irvine, CA 92717.

IEEE Log Number 9200408 deformations on microscopic image resolution is evaluated. From the numerical analysis, it is shown that the resolution degradation due to gradient coil vibrations and deformations could be an important resolution limiting factor in NMR microscopy in addition to other well-known limiting factors such as bandwidth-limited resolution, diffusion-limiting resolution [1], [2], or susceptibility-limited resolution [5].

\section{Finite Element Model of THE GRADIENT CoIL}

The finite element is an efficient approach for numerical analysis of continua. It is based on the discretization of a given structure using a finite number of elements to simplify the solution procedure. In structural applications, approximate values of the stresses and deflections in the structure are obtained.

For an elastic material undergoing small stresses, normal and shearing strains are defined as

$$
\epsilon_{x}=\frac{\partial u}{\partial x}, \quad \epsilon_{y}=\frac{\partial v}{\partial y}, \quad \epsilon_{z}=\frac{\partial w}{\partial z}
$$

$\gamma_{x y}=\frac{\partial u}{\partial y}+\frac{\partial v}{\partial x}, \quad \gamma_{y z}=\frac{\partial v}{\partial z}+\frac{\partial w}{\partial y}, \quad \gamma_{z x}=\frac{\partial w}{\partial x}+\frac{\partial u}{\partial z}$

where $\epsilon_{x}, \epsilon_{y}$, and $\epsilon_{z}$, are normal strains; $\gamma_{x y}, \gamma_{y z}$, and $\gamma_{z x}$, are shearing strains; and $u, v$, and $w$ are deformations along $x, y$, and $z$ directions, respectively.

A body acted upon by external forces may, in general, experience deformation characterized by strain. The six components of stress are related to the six components of strain through a proportionality matrix $[D]$ containing thirty six terms for a general anisotropic material. However, for homogeneous and isotropic materials, we have the following stress-strain relationship

$$
\begin{aligned}
\epsilon_{z} & =\frac{1}{E}\left(\sigma_{x}-\nu\left[\sigma_{y}+\sigma_{z}\right]\right) \\
\epsilon_{y} & =\frac{1}{E}\left(\sigma_{y}-\nu\left[\sigma_{z}+\sigma_{x}\right]\right) \\
\epsilon_{z} & =\frac{1}{E}\left(\sigma_{z}-\nu\left[\sigma_{x}+\sigma_{y}\right]\right)
\end{aligned}
$$

and

$$
\gamma_{x y}=\frac{\tau_{x y}}{G}, \quad \gamma_{y z}=\frac{\tau_{y z}}{G}, \quad \gamma_{z x}=\frac{\tau_{z x}}{G}
$$

where $\sigma_{x}, \sigma_{y}$, and $\sigma_{z}$ are normal stresses; $E$ is the modulus of elasticity; $\nu$ is Poisson's ratio; $\tau_{x y}, \tau_{y z}, \tau_{z x}$ are shearing 
stresses; and $G$ is defined as

$$
G=\frac{E}{2(1+\nu)}
$$

In matrix form, the stress-strain relationship may be written as

$$
\{\epsilon\}=[D]\{\sigma\}
$$

where

$$
\{\epsilon\}=\left\{\begin{array}{c}
\epsilon_{x} \\
\epsilon_{y} \\
\epsilon_{z} \\
\gamma_{x y} \\
\gamma_{y z} \\
\gamma_{z x}
\end{array}\right\} \quad \text { and } \quad\{\sigma\}=\left\{\begin{array}{c}
\sigma_{x} \\
\sigma_{y} \\
\sigma_{z} \\
\tau_{x y} \\
\tau_{y z} \\
\tau_{z x}
\end{array}\right\}
$$

and

$$
\begin{gathered}
{[D]=\frac{1}{E}} \\
\cdot\left[\begin{array}{cccccc}
1 & -\nu & -\nu & 0 & 0 & 0 \\
-\nu & 1 & -\nu & 0 & 0 & 0 \\
-\nu & -\nu & 1 & 0 & 0 & 0 \\
0 & 0 & 0 & 2(1+\nu) & 0 & 0 \\
0 & 0 & 0 & 0 & 2(1+\nu) & 0 \\
0 & 0 & 0 & 0 & 0 & 2(1+\nu)
\end{array}\right]
\end{gathered}
$$

Using the virtual work principle or the potential energy theorem [3], [4], the relation between nodal displacements and static forces at discrete points having a total number of degrees of freedom $n$ may be related by the matrix equation

$$
[K]\{q\}=\{p\}
$$

where $p$ is the generalized load vector $(n \times 1), q$ is the generalized displacement vector $(n \times 1)$, and $[K]$ is the inverse of the $[D]$ matrices, and is referred to as the assembled stiffness matrix $(n \times n)$ relating loads and nodal displacements.

In the case of dynamic loading, inertial forces are also considered in the equilibrium formulation, and the equation of motion is written as

$$
[M]\{\ddot{q}\}+[K]\{q\}=\{p(t)\}
$$

where $\{\ddot{q}\}$ is the second derivative of the generalized displacements, and $[M]$ is the mass matrix of the discretized elements. If damping effects are included, (12) becomes

$$
[M]\{\ddot{q}\}+[C]\{\dot{q}\}+[K]\{q\}=\{p(t)\}
$$

where $\{\dot{q}\}$ is the first derivative of the generalized displacements, and $[C]$ is the damping matrix of the discretized elements.

\section{Evaluation of the Gradient CoIl DEFORMATION AND VIBRATION}

For the Maxwell pair ( $z$ gradient coil) and Golay coils ( $x$ and $y$ gradient coils) mounted on a cylindrical acrylic tube (diameter $=2 \mathrm{~cm}$, thickness $=0.2 \mathrm{~cm}$ ), (13) is numerically evaluated by the program SAP90 [6] using a flatfacet [7] plate/shell element. Total of 864 elements (36 layers with 24 nodes per layer) are considered for the finite element analysis. The material properties used for acrylic are: Young's modulus $=6.9 \times 10^{9} \mathrm{~N} / \mathrm{m}^{2} ;$ mass density $=1.2 \times 10^{3} \mathrm{~N}-$ $\mathrm{s}^{2} / \mathrm{m}^{4}$; and Poisson ratio $=0.24$. The damping ratio is set to 0.035 . Membrane components of the displacements (inplane) as well as the flexural degrees of freedom (out-of-plane displacement and rotations) are considered. The analyzed results are shown in the next section.

For an input current of $250 \mathrm{~A}$ (gradient field $=230 \mathrm{G} / \mathrm{cm}$ ), deformations of the gradient coil frame are first evaluated along the $z$ direction. To simplify the analysis, a single current wire is assumed for the gradient coil, i.e., the input current of $250 \mathrm{~A}$ with one turn is equivalent to $2.5 \mathrm{~A}$ with 100 turns. Two circular clamped supports $z= \pm 1.2 \mathrm{~cm}$ (coil radius $=1 \mathrm{~cm}$ ) are used for boundary conditions as shown in Fig. 1. The radial displacements of the gradient coil frame along the $z$ direction are shown in Fig. 2(a) and (b) for the $z$ gradient coil and the $x-y$ gradient coil, respectively. For the $x-y$ coil, the deformations are evaluated on the surface of the coil frame passing through the middle arcs (solid line), and along the line on the coil frame rotated $90^{\circ}$ from the previous line, thereby crossing none of the active wires (dotted line).

As expected for both the $x-y$ and $z$ gradient coils, the maximum displacements occur at those nodes where the coils are mounted, i.e., the Lorentz force applied regions. For an acrylic coil frame, the magnitudes of the maximum nodal displacements are $4.2 \mu \mathrm{m}$ for the $x-y$ coil frame and $1.1 \mu \mathrm{m}$ for the $z$ coil frame. The deformations in the imaging region are, however, much more serious for the $x-y$ gradient coil, because the net Lorentz forces at the inner most 4 arcs around the imaging region have the same direction. On the other hand, the deformations induced by the $z$ gradient coils are zero at the center plane due to the symmetric squeezing and expanding forces.

Based on these results, the coil deformations will pose problems in obtaining microscopic resolution NMR images. In an intuitive evaluation of the image distortion due to gradient deformations we consider the object being imaged as stationary with respect to the deformed coils. Under this condition, it can be expected that the object will now experience a distorted gradient field so that the obtained image will be distorted the same magnitude as the coil deformation. This effect is studied in detail in the next section.

Another interesting coil deformation is vibration during gradient pulsing. For conventional spin-warp pulse sequence, trapezoidal shaped pulses are usually employed in order to allow the transient responses (mechanical transient responses as well as eddy-current associated electrical transient responses [8], [9]) to die out. It is necessary to investigate the magnitude of these transient responses and how long they will last so 


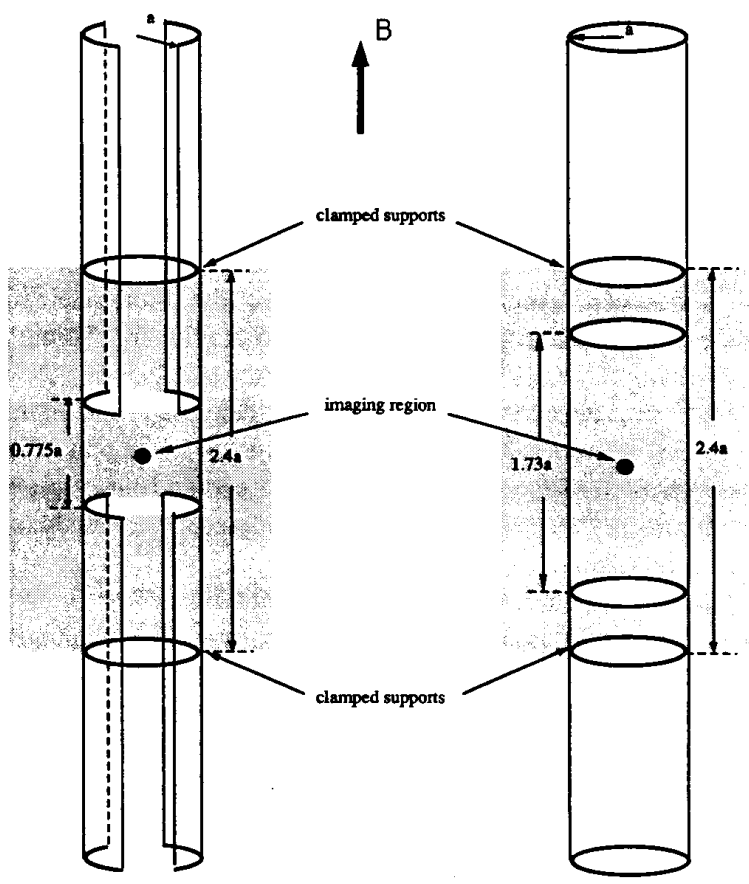

Fig. 1. Geometry of the $x-y$ and $z$ coil frames. (a) $x-y$ coil frame made of four saddle-shaped coils with a distance of $0.755 \mathrm{a}$ between the upper and lower saddle-shaped coils and (b) $z$ coil frame made of a Maxwell pair with a distance of $1.73 \mathrm{a}$ between the two loops where $a=1 \mathrm{~cm}$ is the radius of the coils. The difference in coil frame heights are chosen to satisfy the optimal field linearity constraints. Shaded regions in both coils are the portions used for deformation evaluation. Imaging region is located at the center of the coil frames with a diameter of a few millimeters.

that any further image degradation caused by this effect can be minimized.

For this study, time responses of coil deformations are investigated for three different shapes of input currents with different rising and falling time periods. Only the time responses of the $x-y$ coil nodal displacements subject to these different input gradient pulses are studied. Time responses for the $z$ coil nodal displacements can be similarly obtained. The time response of the nodal displacements subject to trapezoidal-shaped inputs with (a) $0 \mu \mathrm{s}$, (b) $200 \mu \mathrm{s}$ rising and falling time, and (c) sine-shaped input are shown in Fig. 3(a)-(c), respectively. The node is chosen to be on the middle of the coil frame [middle point of the solid line in Fig. 2(b)].

As can be seen from Fig. 3(a), for the rectangular input pulse, large transient response occurred right after the beginning and ending of the pulse. This transient effects last longer than $1 \mathrm{~ms}$. This results in large image distortion and resolution degradation if the data is acquired during that period. These transient effects can be greatly reduced, however, if a trapezoidal input is used. As shown in Fig. 3(b), the peak transient response is reduced by about $95 \%$ compared to Fig. 3(a). As a comparison, for a sinusoidal gradient pulse, the corresponding transient effects is negligible due to its smooth characteristic. These time responses may be useful for designing optimal pulse shapes which induce minimum coil
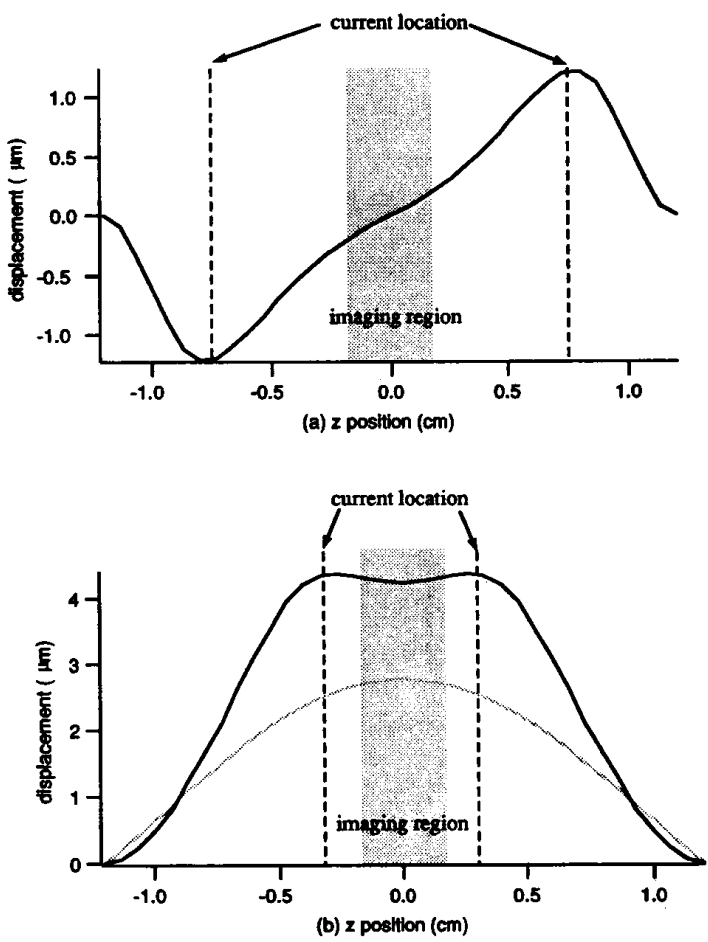

Fig. 2. Nodal displacements of the acrylic coil frames. (a) The displacements of the $z$ gradient coil frame. Note that the deformation is circularly invariant in the Maxwell pair. (b) The displacements of the $x-y$ gradient coil frame.

vibrational motions and deformations.

\section{IMAGE RESOlUtion DEgRADATION DUE TO GRADIENT COIL DEFORMATION}

Distorted gradient fields due to deformed gradient coils cause image distortion. Based on the previous numerical analysis of the gradient coil deformations, the field deformation can be evaluated accordingly. To simplify the analysis, deformed current paths are only considered for further image distortion analysis and other deformations of the gradient coil are not considered. Note that the geometry of the gradient coil and coil frame material are already incorporated in the evaluation of the deformations of the current paths. For the readout gradient, if the vibrations shown in Fig. 3 are sufficiently suppressed by proper design of the input current waveform, then the gradient field deformations are time invariant during the data acquisition period. If the field distortion is represented by $\Delta B$, then the image distortion (displacement) can be expressed as

$$
\Delta x=\frac{\Delta B}{g}
$$

where $g$ is the slope of the gradient field.

Using the Biot-Savart law, image distortions along the $x-y$ and $z$ directions are shown in Fig. 4 for an imaging region of $0.5 \mathrm{~mm}$ (pixel size is $\sim 2 \mu \mathrm{m}$ ). The results for both cases appears to be almost constant, due to the fact that the imaging region is small compared to the coil frame diameter. However, the corresponding image distortion due to the $x-y$ 


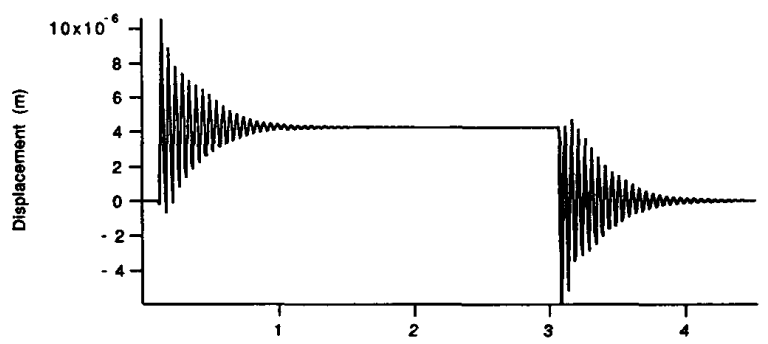

(a) Time (ms)

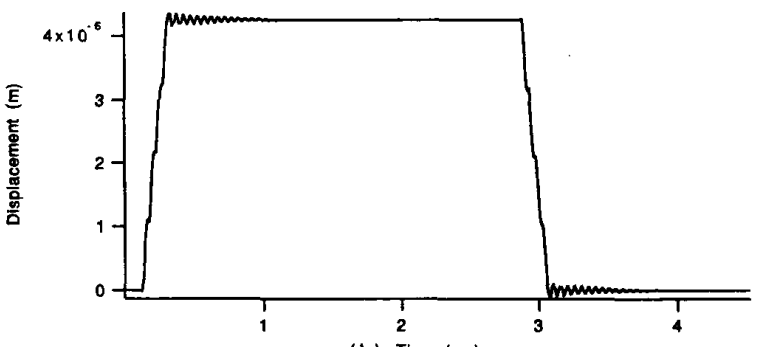

(b) Time (ms)

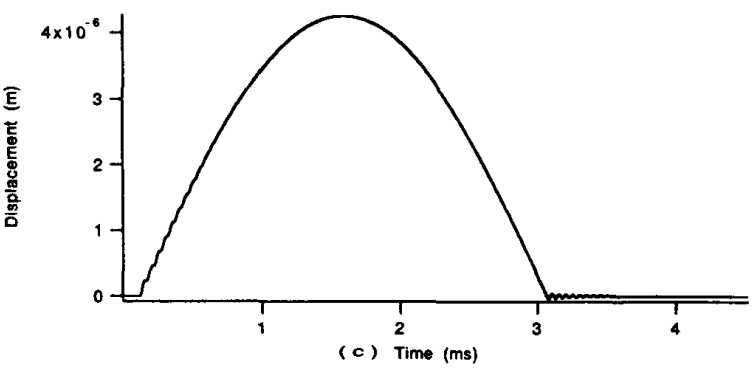

Fig. 3. The time response of the $x-y$ coil nodal displacements subject to three different inputs with trapezoidal-shaped inputs of (a) $0 \mu \mathrm{s}$, and (b) $200 \mu \mathrm{s}$, rising and falling time, and (c) sine-shaped input. The node is chosen to be on the middle of the coil frame.

coil deformation is much bigger than the image distortion due to the $z$ coil deformation. Note that there is no physical deformation at the center plane induced by the $z$ gradient coil because of the opposing Lorentz forces, however, there is image distortion due to the deformed current paths which are not located at the center plane.

Compared to the almost-constant image distortion due to the readout gradient deformation, image distortion by the phase encoding gradient deformation results in much more serious effects because of its time variant characteristics. From the Nyquist sampling theorem, the gradient step can be expressed as

$$
\Delta G=\frac{1}{\gamma X T}
$$

where $\gamma$ is the gyromagnetic ratio, $X$ is the size of the imaging region and $T$ is the duration of the phase encoding pulse.

As (15) implies, $\Delta G$ and thus the coil deformation may be reduced by increasing $T$. However, molecular diffusion also increases in proportion to $T^{3}$ in NMR microscopy [2]. Normally $T$ will not exceed a few milliseconds in these experiments. For $T=2 \mathrm{~ms}$, the required gradient strength and

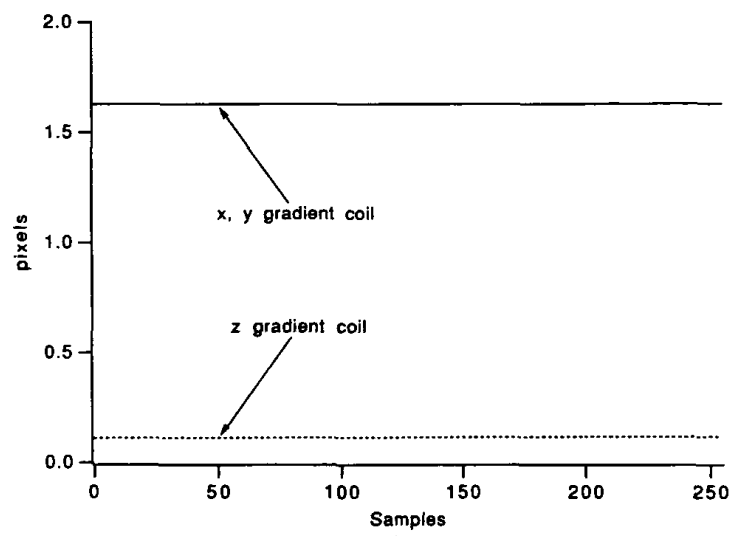

Fig. 4. Image distortions due to the time invariant coil deformations of the readout gradient: (a) $x-y$ coils and (b) $z$ coil deformation. Vertical axis is the image distortion in the unit of pixels. Horizontal axis shows the 256 samples over an imaging region of $0.5 \mathrm{~mm}$ (pixel size is $\sim 2 \mu \mathrm{m}$ ).

thus current intensity for the 256 phase encoding steps of an imaging region of $0.5 \mathrm{~mm}$ varies from $-371 \mathrm{~A}$ to $368 \mathrm{~A}$ with 2.9 A increments. Due to the different current intensity at each step, the coil frame will deform differently depending upon the magnitude and polarity of the input current. This time-variant coil deformation causes time-variant field distortions which in turn result in image blurring and resolution degradation.

A common way to evaluate image blurring and resolution degradation is to measure the full width at half maximum (fwhm) of the point spread function (PSF). Consider a stationary point source, if there is no field distortion throughout the entire data acquisition period, the reconstruct image (PSF) will be a delta function located at the position of the point source. However, if a time dependent field distortion occurs, the reconstructed PSF will no longer be an ideal delta function but a blurred one. This has an effect similar to the diffusion process where the situation is reversed. The gradient fields are unchanged, but the spins are moving throughout the data acquisition periods [2], [10]-[12]. Both cases result in degraded PSF's. By measuring the fwhm value of the deformed PSF, the degree of image blurring and resolution degradation can be assessed.

For a point source located at the center of the imaging region, the corresponding PSF's due to the $x-y$ and $z$ coil deformations for three different image resolutions are shown in Fig. 5(a)-(c) and Fig. 6(a)-(c). Both figures are drawn with zero appending from 256 complex data points to 1024 complex data points and an image resolution of (a) $2 \mu \mathrm{m}$, (b) $4 \mu \mathrm{m}$, and (c) $10 \mu \mathrm{m}$, respectively. Their corresponding fwhm values are listed in Table I.

As can be seen, the PSF degradation due to $z$ coil deformations does not change much from $10 \mu \mathrm{m}$ resolution to $2 \mu \mathrm{m}$ resolution, because of the small opposing coil deformations as mentioned earlier. For the $x-y$ coil deformations, however, the PSF degradation becomes much worse. The fwhm values increase from about 1.0 pixel (real and magnitude) in the $10 \mu \mathrm{m}$ resolution case to $5.0 / 7.5 \mathrm{pixels}$ (real/magnitude) in the $2 \mu \mathrm{m}$ resolution case. This severe PSF degradation may 


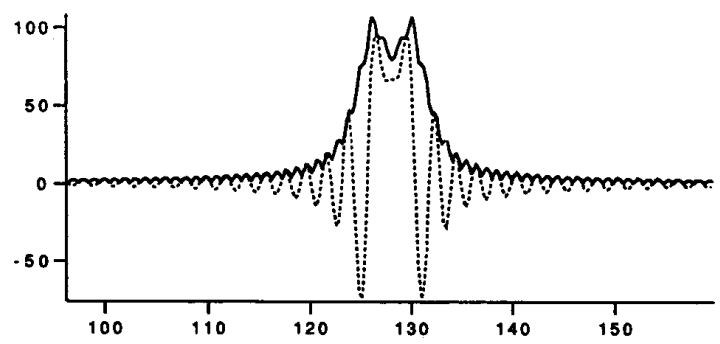

(a)

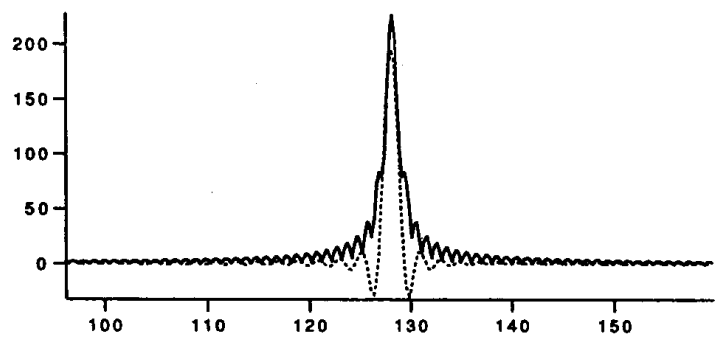

(b)

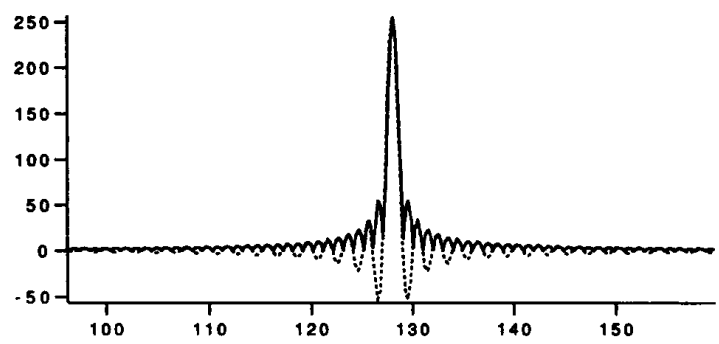

(c)

Fig. 5. Distorted point spread functions (PSF's) due to the time variant phase encoding gradient variation of the $x-y$ coils: with (a) $2 \mu \mathrm{m}$ image resolution, (b) $4 \mu \mathrm{m}$ image resolution, and (c) $10 \mu \mathrm{m}$ image resolution. The dotted curve corresponds to the real part and the solid curve corresponds to the magnitude part of the PSF. Horizontal axis of the plots are drawn with zero appending from 256 complex data points to 1024 complex data points.

be alleviated by either choosing a more rigid material than acrylic or by increasing the phase encoding period $T$, at a cost of increasing diffusion effects as previously reported [2], $[10]-[12]$.

\section{CONCLUSION}

Coils deformations caused by gradient field applications have been studied using finite element analysis. For acrylic coil frames, about $\sim 5 \mu \mathrm{m}$ deformations occur at the coil locations. This magnitude of coil deformation causes a $\sim 2$ pixels image shift along the readout gradient direction for an image resolution of $2 \mu \mathrm{m}$. In the case of the phase encoding direction, however, much more serious resolution degradation occurs due to variation of the gradient field strength. It can be shown that with increased gradient strength, the corresponding PSF degradation will increase exponentially. Increasing gradient duration, instead of increasing gradient strength, may be an alternative to achieve higher resolution without degrading the

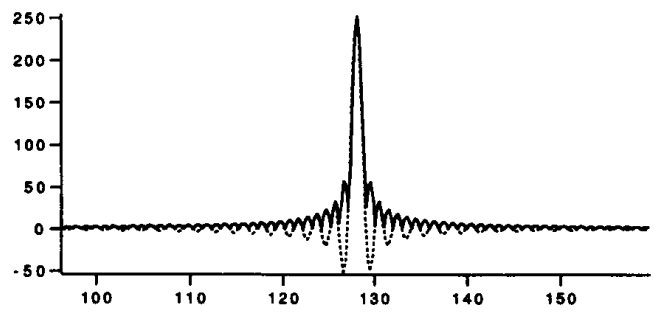

(a)

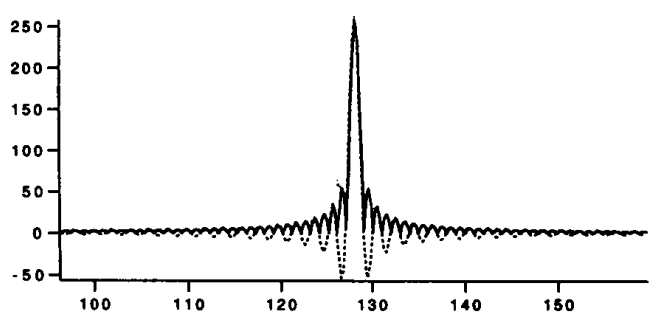

(b)

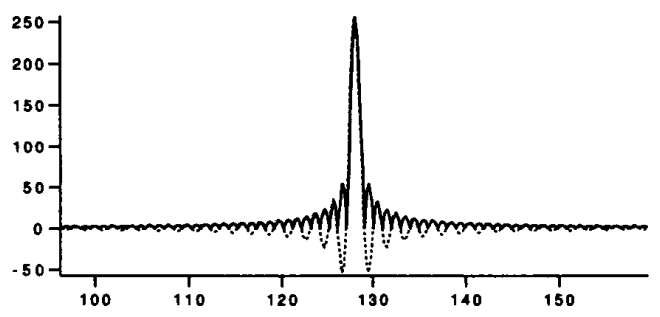

(c)

Fig. 6. Distorted point spread functions (PSF's) due to the time-variant phase encoding gradient variation of the $z$ coil: with (a) $2 \mu \mathrm{m}$ image resolution, (b) $4 \mu \mathrm{m}$ image resolution, and (c) $10 \mu \mathrm{m}$ image resolution. The dotted curve corresponds to the real part and the solid curve corresponds to the magnitude part of the PSF. Horizontal axis of the plots are drawn with zero appending from 256 complex data points to 1024 complex data points.

TABLE I

FWhM Values of the Real and Magnitude Parts of the PSF Degradation Due to the Time Variant Phase Encoding Gradient Variation of the $x-y$ and $z$ Colls Shown in Figs. 5 and 6 . The Phase Encoding Time Period $T$ is Set to 2 ms. All Values are in Pixel Units

\begin{tabular}{cccc}
\hline $\begin{array}{c}\text { image } \\
\text { resolution }\end{array}$ & no deformation & $\begin{array}{c}x-y \text { coil } \\
\text { deformation } \\
\text { real/magnitude }\end{array}$ & $\begin{array}{c}z \text { coil } \\
\text { deformation } \\
\text { real/magnitude }\end{array}$ \\
\hline $2 \mu \mathrm{m}$ & $1.0 / 1.0$ & $5.0 / 7.5$ & $1.0 / 1.1$ \\
$4 \mu \mathrm{m}$ & $1.0 / 1.0$ & $1.5 / 1.6$ & $1.0 / 1.0$ \\
$10 \mu \mathrm{m}$ & $1.0 / 1.0$ & $1.0 / 1.1$ & $1.0 / 1.0$ \\
\hline
\end{tabular}

PSF, however, increasing the gradient duration will increase the diffusion effects.

For acrylic coil frames, the effects of coil deformation and resolution degradation in the phase encoding direction can be ignored if the image resolution is lower than $10 \mu \mathrm{m}$. With enhanced image resolution ( $2 \sim 4 \mu \mathrm{m}$ or above), it is shown that the PSF degradations are so severe that no reasonable image can be produced by increasing the gradient strength alone. Optimal selection of coil frame material together with careful consideration of diffusion effects are necessary for high resolution NMR imaging with resolution beyond $2 \mu \mathrm{m}$. 


\section{ACKNOWLEDGMENT}

The authors would like to thank Computers and Structural, Inc. for its "SAP90" software used for the FEM analysis in this research.

\section{REFERENCES}

[1] Z. H. Cho, C. B. Ahn, S. C. Juh, H. K. Lee, R. E. Jacobs, S. Lee, J.H $\mathrm{Yi}$, and J.M. Jo, "Nuclear magnetic resonance microscopy with $4-\mu \mathrm{m}$ resolution: Theoretical study and experimental results," Med. Phys., vol. 15 , pp. 815,1988

[2] C. B. Ahn and Z.H. Cho, "A generalized formulation of diffusion effects in $\mu \mathrm{m}$ resolution nuclear magnetic resonance imaging," Med. Phys. vol. 16, pp. 22, 1989.

[3] R.D. Cook, Concepts and Applications of Finite Element Analysis. New York: Wiley, 1981, 2nd ed.

[4] O.C. Zienkiewicz, The Finite Element Method. New York: McGrawHill, 1981, 3rd ed.
[5] P. T. Callagham, "Susceptibility-limited resolution in nuclear magnetic resonance microscopy," J. Magn. Reson., vol. 87, pp. 304, 1990.

[6] SAP90-Structural Analysis Programs. Berkeley, CA: Computers and Structures.

[7] W. Weaver, and P. R. Johnston, Finite Elements for Structural Analysis. Englewood Cliffs, NJ: Prentice-Hall, 1984, 2nd ed.

[8] C. B. Ahn and Z. H. Cho, "Analysis of eddy currents in nuclear magnetic resonance imaging," Magn. Reson. Med., vol. 17, pp. 149, 1991.

[9] _- "Analysis of eddy-current induced artifacts and the temporal compensation in nuclear magnetic resonance imaging," IEEE Trans. Med. Imaging, vol. 10, pp. 47, 1991.

[10] C. B. Ahn, S.Y. Lee, O. Nalcioglu, and Z.H. Cho, "An improved nuclear magnetic resonance diffusion coefficient imaging method using an optimized pulse sequence," Med. Phys., vol. 13, pp. 789, 1986.

[11] W.C. Chu, C.B. Ahn, and Z.H. Cho, "Monte Carlo simulation of diffusion in NMR microscopy," Book of Abstract of the 9th Ann. Sci. Meeting Soc. Magn. Reson. Med., New York, p. 394, 1990.

[12] C.B. Ahn, W.C. Chu, and Z.H. Cho, "Imaging strategies in three dimensional nuclear magnetic resolution microscopy,"J. Magn. Reson., to be published. 